\title{
Dynamic Observation of an Atom-Sized Gold Wire by Real-Time Defocus Image Modulation Processing Electron Microscope
}

\author{
Y. Takai, T. Kawasaki and Y. Kimura
}

Department of Material and Life Science, Graduate School of Engineering, Osaka University, 2 -1 Yamada-oka, Suita, Osaka 565-0879, Japan

Spherical aberration-free phase imaging has a high potential to determination localized atomic structures at surfaces and interfaces directly in transmission electron microscopy, and some approaches [1,2] based on image processing have been proposed in this recent decade. We have developed a real-time defocus-image modulation processing electron microscope (DIMP-EM) to observe spherical aberration-free phase and amplitude images separately with a time resolution of $1 / 30^{\text {th }}$ of a second [3]. To realize high resolution phase observation, we newly developed a custom designed, floating type, accelerating voltage generation system [4] which enables to control focus rapidly and precisely at ever 8 micro-seconds, and a unique high-speed image processing CCD video camera [5] which enables addition or subtraction between the two successive images taken within a video field period $(1 / 60 \mathrm{~s})$. In the present paper, we report dynamic observation of an atomsized gold wire in the phase imaging mode using this novel microscope [6].

Figure 1(a) shows a block diagram of the real-time DIMP-EM of the final version. The main column of the DIMP-EM is essentially the same as a commercial type $200 \mathrm{kV}$ field emission electron microscope, HF-2000. In the real-time DIMP-EM, the newly developed accelerating voltage modulation system and the novel CCD video camera with a high density CCD chip controlled by a field programmable gate arrays (FPGA) are incorporated. Figure 1(b) shows the specific weight function to reconstruct spherical aberration-free phase image using through-focus images and an equivalent modulation function for the accelerating voltage to perform realtime DIMP. The first half of a video field is assigned to take an image with the positive weight and the latter half to take an image with the negative weight. The FPGA, first, controls the vertical charge transfer lines of the chip and stores two sets of the images. Next, the FPGA controls the horizontal charge transfer lines to read out these two successive images and finally, the subtracted image, that is, the spherical aberration-free phase image, is continuously output as an NTSC video signal. The size of the image is $640 \mathrm{x} 480$ pixels. Figure 2 shows the dynamic movement of the atom-sized wire that is continuously observed in spherical aberration-free phase imaging mode by the real-time DIMP-EM. In the images of Figs. 2(a) and 2(c), the right-hand connecting position of the wire moves down by a distance of $1 / 2[110]$ as the two crystals on both sides gradually change their relative positions. The central white dot of the wire shifts to the right in Fig. 2(d) and shifts to the left in Fig. 2(e), indicating that the center atom is maintaining a normal atomic distance in the bulk crystal when the two crystals gradually separate. Therefore, the atom-sized gold wire observed in Fig. 2 might form a crystal slab consisting of a few atoms in depth, because the wire still has the characteristics of gold bulk crystal. It should be noted that the contrast of the wire image is rather strong with a relatively good signal to noise ratio even when the wire consists of a very small number of atoms. A real-time DIMP-EM is now providing a unique approach for studying atomic-scale dynamic phenomena that occur on surfaces and interfaces with high contrast and high resolution that is comparable with that of 1 MV HRTEM. It may also be effective in applications requiring high-resolution observation of biological specimens, which is now under investigation. 
References

[1] T. Ikuta: J. Electron Microsc. 38 (1989) 415

[2] M. OP de Beek, D. Van Dyck and W. Coene: Ultramicroscopy 64 (1996) 167.

[3] Y. Takai, Y. Kimura, T. Ikuta, R. Shimizu et al., J. Electron Microsc. 48 (1999) 879.

[4] Y. Kimura, Y. Takai, T. Kawasaki, R. Shimizu et al., J Electron Microsc. 48 (1999) 873.

[5] K. Nishikata, Y. Kimura, Y. Takai, R. Shimizu, T. Ikuta et al., Optik 112 (2001) 97.

[6] Y. Takai, T. Kawasaki, Y. Kimura, T. Ikuta and R. Shimizu,Phys. Rev. Lett. 87 (2001) 106105.

(a)
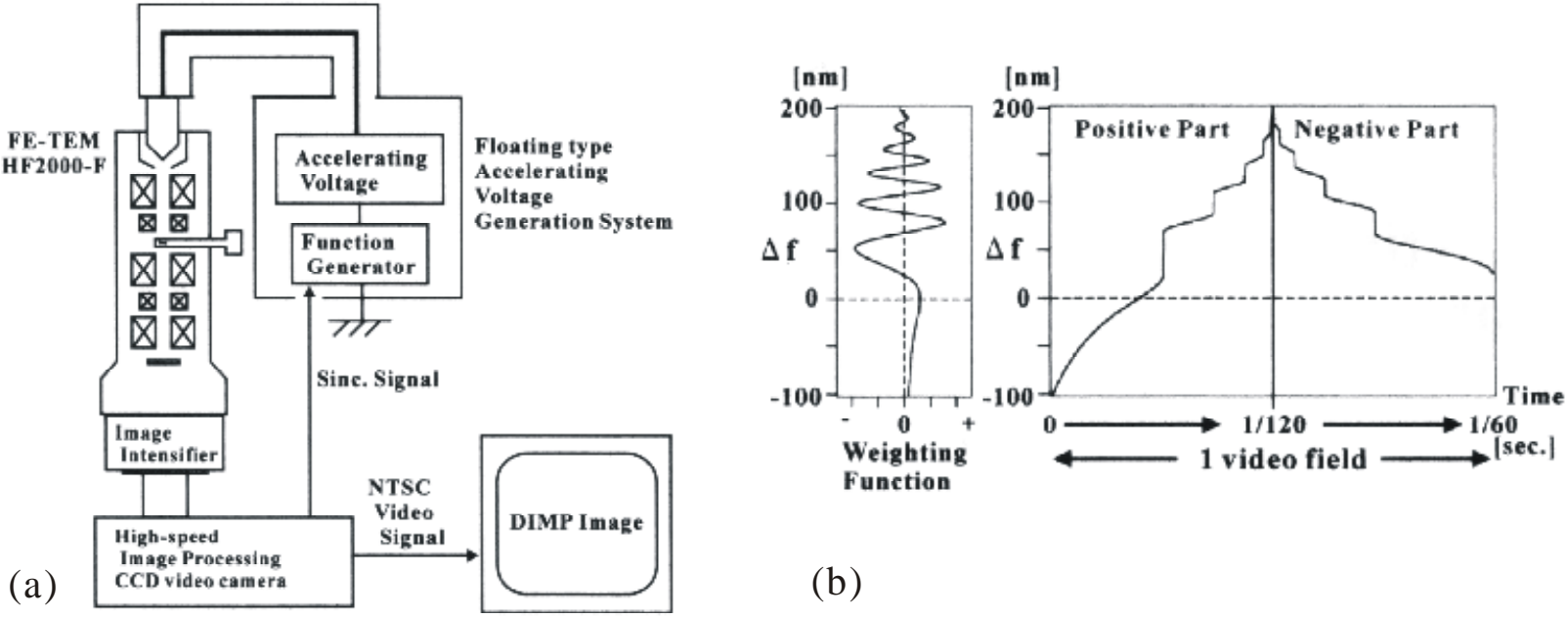

Fig. 1. (a) Block diagram of real-time DIMP-EM. (b) Specific weight function and equivalent modulation function of defocus for performing realtime DIMP.
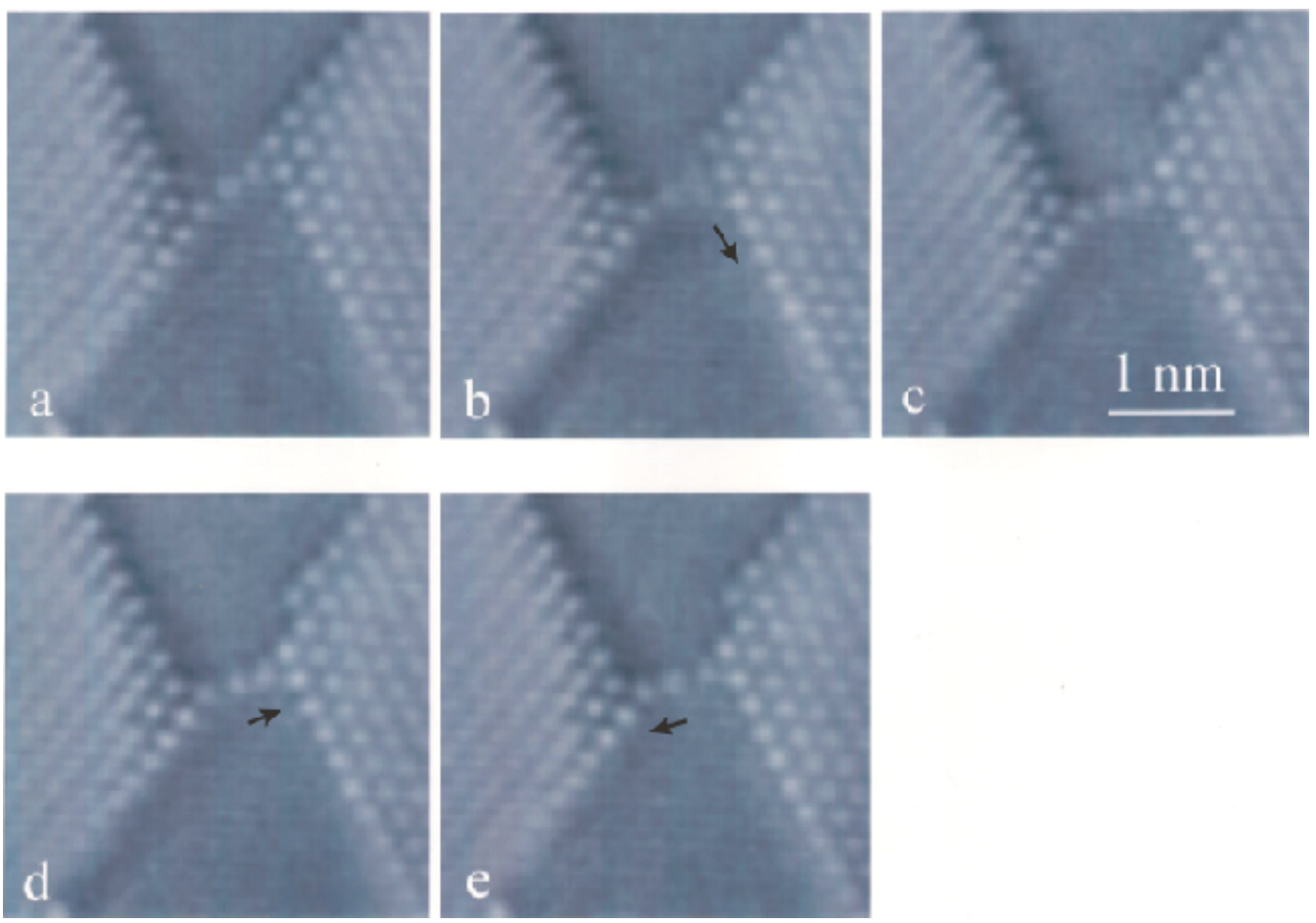

Fig. 2. Movement of atom-sized gold wire:(a) $0.00 \mathrm{~s}$, (b) $0.27 \mathrm{~s}$, (c) $0.40 \mathrm{~s}$, (d) $0.67 \mathrm{~s}$, and (e) $0.80 \mathrm{~s}$. 